Márcia Aires Rodrigues de Freitas'

Angélica Lemos Debs Diniz ${ }^{2}$

Maria Céla dos Santos ${ }^{3}$

Ben Hur Braga Taliberti ${ }^{4}$

ROBERTO RANZA ${ }^{5}$

Artigo original

Palavras-chave

Artéria oftálmica/ultrassonografia

Fluxometria por laser-doppler

Velocidade do fluxo sanguíneo

Lúpus eritematoso sistêmico

Gravidez

Keywords

Ophthalmic artery/ultrasonography Laser-doppler flowmetry

Blood flow velocity

Systemic lupus erythematosus

Pregnancy

\title{
Hiperperfusão no território orbital de gestantes portadoras de lúpus eritematoso sistêmico
}

\author{
Orbital territory byperperfusion in pregnant women with systemic \\ lupus erythematosus
}

\section{Resumo}

OBJETIVO: analisar o comportamento da artéria oftálmica em grávidas portadoras de lúpus eritematoso sistêmico (GL), sem doença renal em atividade, comparando com não-grávidas com lúpus (NGL), sem doença renal em atividade e grávidas normais (GN). MÉTODOS: estudo observacional que analisou as variáveis doplervelocimétricas da artéria oftálmica de $20 \mathrm{GN}, 10 \mathrm{GL}$ e 17 NGL. As variáveis analisadas foram os índices de pulsatilidade (IP), a velocidade diastólica final (VDF) e a razão entre picos de velocidade (RPV). Foram calculadas as médias dos índices e respectivos desvios padrões. Para comparação das médias dos índices dos três grupos, utilizou-se o teste de variância (ANOVA) e prova pós-análise de Tukey, com intervalo de confiança de 95\% (p<0,05). RESULTADOS: o grupo de gestante normal apresentou as seguintes médias e desvio padrão dos parâmetros da artéria oftálmica: $\mathbb{P}=2,4 \pm 0,3 ; R P V=0,5 \pm 0,1$ e $V D F=5,1 \pm 2,1 \mathrm{~cm} / \mathrm{seg}$. Já os dois grupos GL e NGL mostraram, respectivamente, as seguintes médias e desvio padrão

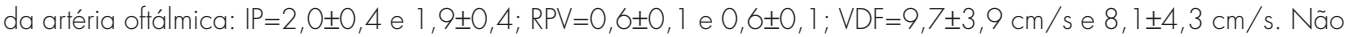
houve diferenças estatísticas significativas quando comparadas as médias do IP, VDF e RPV entre os grupos GL e NGL. Porém, observaram-se diferenças estatísticas significativas entre as médias do IP, VDF e RPV dos grupos GN e GL, com valores mais elevados de VDF e RPV no grupo GL. CONCLUSÕES: houve redução da impedância vascular da artéria oftálmica e hiperperfusão orbital nos dois grupos de pacientes com lúpus em relação às grávidas normais. Os conhecimentos obtidos neste estudo poderão auxiliar no melhor entendimento da fisiopatologia do lúpus eritematoso sistêmico, bem como poderá ser empregado em estudos futuros como método complementar para o diagnóstico diferencial entre a pré-eclâmpsia e a atividade de doença renal nas gestantes com lúpus.

\section{Abstract}

PURPOSE: to analyze the ophthalmic artery functioning in pregnant women with systemic lupus erythematosus (PL) without active renal disease as compared to non-pregnant women with lupus (NPL) without active renal disease, and to normal pregnant women (PN). METHODS: observational study that analyzed ophthalmic artery dopplervelocimetric variables of 20 PN, 10 PL and 17 NPL women. The variables analyzed were: pulsatility index (PI), final diastolic velocity (FDV) and velocity peak ratio (VPR). Mean and standard deviation of these indexes were calculated. For group mean comparison, analysis of variance (ANOVA) and the post-hoc Tukey test have been used, with confidence interval of $95 \%$ ( $p<0.05)$. RESULTS: the PN group showed the following means and standard deviations of ophthalmic artery parameters: $\mathrm{Pl}=2,4 \pm 0,3 ; \mathrm{VPR}=0,5 \pm 0,1$ e FDV $=5,1 \pm 2,1 \mathrm{~cm} / \mathrm{s}$. The PL and NPL groups showed the following values, respectively: $P I=2,0 \pm 0,4$ and $1,9 \pm 0,4 ; V P R=0,6 \pm 0,1$ and $0,6 \pm 0,1 ; F D V=9,7 \pm 3,9 \mathrm{~cm} / \mathrm{s}$ and $8,1 \pm 4,3 \mathrm{~cm} / \mathrm{s}$. There was not significant mean difference between the PL and NPL groups for PI, VPR or FDV. However, statistically significant mean differences were observed between PN and PL for PI, VPR and FDV, with higher values of FDV and VPR in the PL group. CONCLUSIONS: there was a reduction of ophthalmic artery vascular impedance with orbital hyperfusion in the two groups of women with lupus erythematosus as compared to normal pregnant women. These results may help to improve the understanding on pathophysiology of systemic lupus erythematosus. In addition, the present method may be applied in future studies as a complementary procedure for the differential diagnosis between pre-eclampsia and renal failure due to lupus.

Correspondência:

Mária Aires Rodrigues de Freitos Rua Professor Pedro Bernardo, 40, apto 704 - Centro CEP 38400-172 - Uberlândia (MG), Brasi Fone/Fax: 34-32289000 E-mail: mairesfreitas@yahoo.com.br

Recebido

$18 / 9 / 09$
Serviço de Obstetrícia e Ginecologia do Hospital das Clínicas da Universidade Federal de Uberlândia - UFU - Uberlândia (MG), Brasil. ' Pós-graduanda (Mestrado) em Ciências da Saúde da Universidade Federal de Uberlândia - UFU - Uberlândia (MG), Brasil.

2 Professora da Pós-graduação em Ciências da Saúde da Universidade Federal de Uberlândia - UFU - Uberlândia (MG), Brasil. 3 Professora Titular do Departamento de Tocoginecologia da Universidade Federal de Uberlândia - UFU - Uberlândia (MG), Brasil. 4 Professor Titular do Departamento de Clínica Médica da Universidade Federal de Uberlândia - UFU - Uberlândia (MG), Brasil. ${ }^{5}$ Médico Assistente do Departamento de Reumatologia do Hospital das Clínicas da Universidade Federal de Uberlândia - UFU Uberlândia (MG), Brasil. 


\section{Introdução}

O lúpus eritematoso sistêmico (LES) é uma desordem autoimune na qual há produção exacerbada de autoanticorpos e imunocomplexos dirigidos, principalmente contra componentes nucleares, resultando em lesão tecidual progressiva. Os complexos imunes são depositados nos vasos sanguíneos e capilares, causando inflamação local e grande parte das manifestações clínicas da doença. O estímulo responsável por esta alteração imunológica é desconhecido, mas acredita-se que seja decorrente de associação entre fatores genéticos, ambientais e hormonais ${ }^{1,2}$. No período gestacional, a doença está associada a um risco maior de complicações materno-fetais ${ }^{3}$.

A doença atinge em maior número as mulheres, principalmente entre 15 e 35 anos. Como a fertilidade, em geral, não é afetada pela enfermidade, a gravidez associada ao LES é evento comum. A incidência dessa doença entre as gestantes varia de 1 para 660 a $2.952^{4}$.

Durante o pré-natal, há dificuldades no acompanhamento clínico de gestantes com LES, uma vez que algumas alterações podem ser confundidas com a atividade da doença. A lombalgia, o leve edema de joelhos, a alopécia e o cloasma gravídico podem ocorrer na gestação, o que não significa que a doença esteja em atividade ${ }^{4}$. Pode-se deparar com paciente já portadora de hipertensão arterial e proteinúria prévia, com piora clínica após a $24^{\mathrm{a}}$ semana de gestação, o que complica a caracterização precisa da pré-eclâmpsia, influenciando diretamente as condutas a serem tomadas.

A diferenciação entre a nefrite lúpica, a pré-eclâmpsia e as mudanças fisiológicas relacionadas à gestação constituem desafio frequente para o obstetra durante o pré-natal, pois tais situações podem envolver hipertensão, proteinúria, edema e alterações da função renal ${ }^{3-5}$. Apesar de determinadas características clínicas e laboratoriais serem úteis na diferenciação da atividade do lúpus em relação a outras enfermidades renais, muitas vezes temos que recorrer à biópsia renal para definição do diagnóstico. Portanto, torna-se necessário o emprego de métodos diagnósticos mais acurados e pouco invasivos que auxiliem na diferenciação entre a atividade lúpica e a pré-eclâmpsia grave.

A análise dos vasos orbitais vem ganhando destaque no contexto da medicina moderna, tanto na Oftalmologia quanto na Obstetrícia. Esta técnica, além de tornar factível a avaliação de gestantes que apresentam doenças sistêmicas com repercussões no território vascular central, pode ser utilizada no estudo da ação de drogas vasoativas ${ }^{6}$. $\mathrm{O}$ aprimoramento desta técnica permitiu o estudo do comportamento hemodinâmico das artérias do sistema nervoso central pela análise das artérias retrobulbares. Isto só foi possível devido às similaridades embriológicas, anatômicas e funcionais da artéria oftálmica com os vasos de pequeno calibre do sistema nervoso central. Visto que a artéria oftálmica dá origem aos vasos que formam a circulação orbital, ela é o principal vaso utilizado no estudo Doppler do leito orbital ${ }^{7}$.

A doplervelocimetria da artéria oftálmica é um método não-invasivo cujo emprego em gestantes foi descrito em 1995, em estudo no qual os autores analisaram um grupo de pacientes com pré-eclâmpsia ${ }^{8}$. Os autores identificaram redução importante nos índices de pulsatilidade (IP) desta artéria associada à presença de hiperperfusão orbital, contrariando a hipótese fisiopatológica clássica de vasoconstricção sistêmica nesta doença. A partir dessa publicação, vários autores identificaram o mesmo padrão de baixa impedância na artéria oftálmica no grupo de gestantes com pré-eclâmpsia-14. Diferentes variáveis Doppler foram estudadas nos últimos anos, sendo a mais acurada na identificação das formas graves de pré-eclâmpsia, a razão entre picos de velocidade (RPV) $)^{11,13,14}$. Além disso, estão descritos na literatura os parâmetros de normalidade das variáveis Doppler da artéria oftálmica de gestantes normais ${ }^{15,16}$.

O interesse relativo ao estudo Doppler da artéria oftálmica em Obstetrícia baseia-se na capacidade do método auxiliar ao diagnóstico diferencial de casos suspeitos de pré-eclâmpsia em pacientes com proteinúria ou hipertensão crônica pré-instalada. Portanto, o método poderá ser de grande valor na diferenciação dos quadros hipertensivos durante a gestação de pacientes com LES. Para isso, tornase importante estabelecer o padrão vascular da artéria oftálmica em pacientes com LES sem pré-eclâmpsia.

Ainda não está estabelecido, na literatura, se o LES altera a resistência vascular de artérias retrobulbares. Nos vasos sanguíneos, o LES está associado à aterosclerose acelerada, vasculites diversas e fenônemos trombóticos ${ }^{2,17}$. É desconhecido o mecanismo de autorregulação e a resposta à produção de óxido nítrico e prostaciclina em vasos sanguíneos lesados pela doença ${ }^{18}$.

O objetivo deste estudo foi analisar o comportamento da artéria oftálmica em grávidas portadoras de LES e sem doença renal em atividade, em relação às mulheres não-grávidas com LES, sem doença renal em atividade e grávidas normais.

\section{Métodos}

Foi realizado estudo observacional no qual se analisou os índices doplervelocimétricos da artéria oftálmica de três grupos de pacientes: Grupo Grávidas Normais (GN), constituído de 20 pacientes, Grupo Grávidas com LES (GL), com 10 grávidas portadoras de LES sem atividade de doença renal, e Grupo de Não-grávidas com LES (NGL), constituído por 17 mulheres com LES sem doença renal em atividade. As pacientes foram selecionadas nos Ambulatórios 
de Gestação de Alto Risco e/ou Reumatologia e Gestação de baixo risco do Hospital das Clínicas da Universidade Federal de Uberlândia (UFU) no período de janeiro de 2007 a dezembro de 2008.

O projeto de pesquisa foi aprovado pelo Comitê de Ética em Pesquisa da UFU por meio do parecer $n^{\circ}$ 201/08. Todas as pacientes assinaram o termo de consentimento livre e esclarecido.

O diagnóstico de LES se fundamentou na presença de pelo menos quatro dos 11 critérios estabelecidos pelo American Rheumatism Association ${ }^{19}$, em 1982, em qualquer fase de evolução da doença, utilizados pela equipe médica do Ambulatório de Reumatologia do Hospital das Clínicas da UFU (Quadro 1).

Os critérios de inclusão estabelecidos para o Grupo Grávidas Normais foram: ausência de doenças maternas sistêmicas, níveis pressóricos normais, ausência de proteinúria e cilindrúria no exame de urina de rotina, gestação única, feto vivo e sem anomalias estruturais, idade gestacional acima de 20 semanas, calculada com base na ultrassonografia realizada no primeiro trimestre independentemente da data da última menstruação relatada pela gestante. Os critérios de inclusão utilizados para o grupo de grávidas com LES foram: pacientes com quatro ou mais critérios de classificação do LES em qualquer fase de evolução da doença, níveis pressóricos normais, ausência de proteinúria e cilindrúria no exame de urina de rotina, gestação única, feto vivo e sem anomalias estruturais, idade gestacional acima de 20 semanas, calculada com base na ultrassonografia realizada no primeiro trimestre independentemente da data da última menstruação relatada pela grávida.

No Grupo Não-grávidas com LES, foram incluídas pacientes na menacme que relataram ciclos menstruais regulares há pelo menos seis meses antes do início do estudo, com quatro ou mais critérios de classificação do LES em qualquer fase de evolução da doença, níveis pressóricos normais e ausência de proteinúria, hematúria, cilindrúria no exame de urina de rotina. Os três grupos estudados foram considerados homogêneos quanto à idade materna, idade gestacional e ao tempo de evolução do lúpus. Não houve diferenças significativas entre as médias dos grupos. As características dos três grupos estudados estão descritas na Tabela 1.

Os critérios de exclusão para os três grupos foram: diabetes mellitus ou gestacional, sinais clínicos de estenose da artéria carótida interna; doença ocular vascular obstrutiva (a trombose da veia central da retina, a trombose do ramo venoso, a oclusão da artéria central da retina ou a oclusão do ramo arterial); cirurgia ocular prévia; cardiopatias que poderiam alterar o ritmo cardíaco; tabagismo; uso de drogas ilícitas. Foram também excluídas pacientes com doença renal em atividade definida por: proteinúria maior que $500 \mathrm{mg} /$ dia ou 3+ no exame de urina e presença de cilindros celulares hemáticos, leucócitos ou mistos.

Analisaram-se amostras de urina em tira reagente de todas as pacientes do estudo. O método laboratorial para avaliação semiquantitativa de proteinúria e cilindrúria foi o uso das tiras para teste múltiplos da marca Bayer Multistix 10 SG, United Kingdown, Grã-Bretanha, com realização de leitura eletrônica. Os níveis pressóricos foram aferidos com as pacientes sentadas, aplicando-se o aparelho com manguito de $13 \mathrm{~cm}$ no membro superior direito e mantendo o mesmo elevado na altura do precórdio. A pressão diastólica foi determinada pelo quinto ruído de Korotkoff correspondente ao desaparecimento da bulha cardíaca. Foi considerado o limite superior da normalidade os níveis pressóricos de 139/89 mmHg, o que corresponde à pressão arterial média de 105,6 mmHg.

Para a realização do estudo Doppler das artérias oftálmicas, foi utilizado equipamento Sonoace 8800 SA Live da Medison ${ }^{\circledR}$, Seoul, South Korea e transdutor linear na frequência de 10 $\mathrm{MHz}$, segundo técnica já descrita na literatura ${ }^{20}$.

Quadro 1 - Critérios de classificação do lúpus eritematoso sistêmico proposto pelo American College of Rheumatology, estabelecidos em 1982 e revisados em 1997

\begin{tabular}{|c|c|}
\hline Critérios & Descrição dos criférios do lúpus erifemałoso sistêmico \\
\hline Erifema malar & Lesão eritematosa fixa em região malar, plana ou em relevo \\
\hline Lesão discoide & Lesão eritematosa, infiltrada, com escamas queratócitas aderidas e tampões foliculares, que evolui com cicatriz atrófica e discromia \\
\hline Fotossensibilidade & Exantema cutâneo com reação não-usual à exposição à luz solar, de acordo com história do paciente ou com o observado pelo médico \\
\hline Úlceras orais/nasais & Úlceras orais ou nasofaríngeas, usualmente indolores, observadas pelo médico \\
\hline Artrite & Não-erosiva, usualmente envolvendo duas ou mais articulações periféricas, caracterizada por dor e edema ou derrame articular \\
\hline Serosite & $\begin{array}{l}\text { Pleuris (caracterizada por história convincente de dor pleurítica, atrito auscultado pelo médico ou evidência de derrame pleural) ou pericardite } \\
\text { (documentada por eletrocardiograma, atrito, ou evidência de derrame pericárdico) }\end{array}$ \\
\hline Comprometimento renal & Proteinúria (> de 500 mg/dia ou 3+) ou cilindrúria anormal (presença de cilindros hemáticos, tubulares, granulosos ou mistos) \\
\hline Alterações neurológicas & Convulsões (na ausência de outras causas) ou psicose (na ausência de outra causa) \\
\hline Alterações hematológicas & $\begin{array}{l}\text { Anemia hemolítica ou leucopenia (menor que } 4.000 \mathrm{~mm}^{3} \text { em duas ocasiões ou mais) ou linfopenia (menor que } 1.500 \mathrm{~mm}^{3} \text { em duas ocasiões ou mais) } \\
\text { ou plaquetopenia (menor que } 100.000 / \mathrm{mm}^{3} \text { na ausência de outra causa) }\end{array}$ \\
\hline Alterações imunológicas & $\begin{array}{l}\text { Anticorpo anti-DNA nativo ou anti-Sm ou presença de anticorpo antifosfolipídeo com base em: a) níveis anormais de IGG e IGM anticardiolipina; b) teste positivo para } \\
\text { anticoagulante lúpico; ou c) teste falso positivo para sífilis por no mínimo seis meses }\end{array}$ \\
\hline Anticorpos antinucleares & $\begin{array}{l}\text { Título anormal de anticorpo antinuclear por imunofluorescência indireta ou método equivalente, em qualquer época, e na ausência de drogas conhecidas por estarem } \\
\text { associadas à síndrome de lúpus induzido por drogas }\end{array}$ \\
\hline
\end{tabular}


O exame foi realizado com a paciente em decúbito dorsal, após dez minutos de repouso, sempre pelo mesmo examinador. $\mathrm{O}$ transdutor era posicionado transversalmente sobre a pálpebra superior, com os olhos fechados, após a colocação de uma pequena quantidade de gel. Foram realizados movimentos em sentido crânio-caudal, identificando-se o vaso sem pressionar o transdutor sobre a pálpebra, a fim de evitar alterações dos dados obtidos. A artéria oftálmica era observada medialmente ao nervo óptico e seu fluxo, registrado a $15 \mathrm{~mm}$ do disco óptico. Após a identificação da artéria, foram registradas seis ondas sem mudanças do padrão para posterior estudo em uma onda. O local de avaliação da artéria era sempre o mesmo para cada sujeito da pesquisa. A aferição dos índices foi efetuada em apenas um olho, uma vez comprovada a semelhança entre ambos ${ }^{14,21}$. O ângulo de insonação da amostra de volume da dopplervelocimetria foi abaixo de 20 graus, com filtro de $50 \mathrm{HZ}$, frequência de repetição de pulsos de $125 \mathrm{kHZ}$ e amostra volume de $2 \mathrm{~mm}$.

As pacientes foram submetidas à avaliação da artéria oftálmica, com registro das seguintes variáveis de interesse: índice de pulsatilidade, pico de velocidade mesodiastólica (P2) e velocidade diastólica final (VDF). O pico de velocidade mesodiastólico foi calculado pela medida da primeira elevação diastólica da onda de velocidade do fluxo, expressa em centímetros por segundo $(\mathrm{cm} / \mathrm{s})$. A razão entre picos de velocidade foi determinada a partir da seguinte fórmula: $\mathrm{RPV}=\mathrm{P} 2 / \mathrm{P} 1$, onde $\mathrm{P} 1$ representa o pico de velocidade sistólica e P2 o pico de velocidade mesodiastólica após a incisura protodiastólica.

Os três grupos estudados foram pareados quanto à idade materna, idade gestacional e tempo de evolução do lúpus. Para tanto, foi aplicado o teste $t$ de Student, comparando-se a série de valores duas a duas. Foram calculadas as médias e desvios padrões do IP, VDF e RPV da artéria oftálmica nos três grupos de pacientes. Para comparação entre as médias dos índices dos três grupos, utilizou-se o teste de variância (ANOVA) e prova pós-análise de Tukey, com intervalo de confiança de $95 \%(\mathrm{p}<0,05)$.

\section{Resultados}

Não houve diferenças significativas entre as médias dos grupos, o que confirma a homogeneidade da amostra quanto aos fatores estudados. As características dos três grupos estudados estão descritas na Tabela 2.

O Grupo Gestantes Normais apresentou as seguintes médias e desvios padrões dos parâmetros da artéria oftálmica: $\mathrm{IP}=2,4 \pm 0,3 ; \mathrm{RPV}=0,5 \pm 0,1$ e VDF= $=5,1 \pm 2,1 \mathrm{~cm} / \mathrm{seg}$. Já os dois grupos de gestantes com lúpus e mulheres não-gestantes com lúpus mostraram, respectivamente, as seguintes médias e desvios padrões da artéria oftálmica: IP $=2,0 \pm 0,4$ e $1,9 \pm 0,4 ; \mathrm{RPV}=0,6 \pm 0,1$ e $0,6 \pm 0,1 ; \mathrm{VDF}=0,9 \pm 3,9 \mathrm{~cm} / \mathrm{s} \mathrm{e}$ $8,1 \pm 4,3 \mathrm{~cm} / \mathrm{s}$ (Tabela 2).
Tabela 1 - Média e desvio padrão do IP, RPV e VDF das artérias oftálmicas analisadas nos três grupos de pacientes

\begin{tabular}{lccc}
\hline Grupos & IP* $^{*}$ & RPV* $^{*}$ & VDF* $^{*}(\mathrm{~cm} / \mathrm{seg})$ \\
\hline Normal & $2,4 \pm 0,3$ & $0,5 \pm 0,1$ & $5,1 \pm 2,1$ \\
Gestantes com lúpus & $2,0 \pm 0,4$ & $0,6 \pm 0,1$ & $9,7 \pm 3,9$ \\
Mulheres com lúpus & $1,9 \pm 0,4$ & $0,6 \pm 0,1$ & $8,1 \pm 4,3$ \\
\hline
\end{tabular}

*Média \pm desvio padrão.

IP: índice de pulsatilidade; RPV: razão entre picos de velocidade; VDF: velocidade diastólica final.

Tabela 2 - Características demográficas dos três grupos de pacientes analisados

\begin{tabular}{lccc}
\hline & $\begin{array}{c}\text { Grávidas } \\
\text { normais }(\mathrm{n}=20)\end{array}$ & $\begin{array}{c}\text { Grávidas com } \\
\text { lúpus }(\mathrm{n}=10)\end{array}$ & $\begin{array}{c}\text { Mulheres com } \\
\text { lúpus }(\mathrm{n}=17)\end{array}$ \\
\hline Idade (anos) & $29,1 \pm 2,8$ & $25,3 \pm 6,2$ & $30,2 \pm 5,9$ \\
Idade gestacional (semanas) & $29,4 \pm 6$ & $30,0 \pm 5$ & - \\
\hline Tempo de diagnóstico do lúpus & - & $4,8 \pm 1,8$ & $5,7 \pm 4,7$ \\
\hline
\end{tabular}

Não houve diferenças estatísticas significativas quando comparadas as médias do IP, VDF e RPV entre os grupos de pacientes com LES grávidas ou não-grávidas. Porém, foram observadas diferenças estatísticas significativas entre as médias do IP, VDF e RPV dos grupos de grávidas normais e grávidas com LES, com valores menores de IP e maiores de VDF e RPV no grupo de grávidas com LES, sendo a probabilidade para estas variáveis inferior a 0,05 .

A razão entre picos de velocidade e a VDF apresentaram valores maiores no grupo de gestantes com LES em relação ao grupo das gestantes normais, bem como o IP apresentou-se reduzido no grupo de gestantes com LES em relação ao grupo normal, demonstrando sinais de hiperperfusão e queda da impedância no território orbital de pacientes gestantes com LES.

\section{Discussão}

O lúpus eritematoso sistêmico é uma entidade clínica complexa que, quando coexiste com a gestação, pode levar a complicações no binômio materno-fetal. Além disso, sabe-se que o acompanhamento pré-natal torna-se mais difícil devido às modificações fisiológicas da gestação que, muitas vezes, se confundem com os sinais de exacerbação da doença materna ${ }^{3-5}$.

Apesar das dificuldades encontradas para a caracterização da atividade lúpica na gravidez, existem parâmetros clínicos e laboratoriais que auxiliam no seu diagnóstico. São sinais altamente preditivos de nefrite lúpica o consumo de frações do complemento, como C3 e C4, a positividade e ascensão de títulos de anticorpos anti-DNA nativo, além das evidências clínicas de LES extrarrenal como artrite, vasculite cutânea, úlceras orais e linfadenopatias em associação a sedimento urinário com presença de leucócitos, hemácias e cilindros hemáticos. A pré-eclâmpsia é sugerida pelo aumento 
de ácido úrico e enzimas hepáticas e pela ausência de cilindro hemáticos, leucocitários, mistos no exame de urina de rotina 5 .

A presença de hipertensão e proteinúria de início súbito após a $24^{\mathrm{a}}$ semana de gestação pode caracterizar o quadro de pré-eclâmpsia ${ }^{22}$. Porém, se a paciente já tem proteinúria devido à nefrite lúpica e apresenta piora do quadro hipertensivo ao longo da gestação, o diagnóstico da pré-eclâmpsia pode ser dificultado, influenciando diretamente no prognóstico materno-fetal. Portanto, torna-se importante a descrição de novos parâmetros que poderão ser empregados na rotina de diagnóstico diferencial nas gestantes com LES.

O exame Doppler dos vasos orbitais permite definir o comportamento hemodinâmico dos vasos sanguíneos do compartimento cerebral. Isto só é possível devido às similaridades embriológicas, anatômicas e funcionais da artéria oftálmica com os vasos de pequeno calibre do sistema nervoso central. A artéria oftálmica dá origem aos demais vasos que formam a circulação orbital, o que a torna o principal vaso arterial utilizado no estudo do território orbital ${ }^{8-11}$

O estudo Doppler da artéria oftálmica é descrito na literatura como exame simples, não-invasivo e de fácil execução ${ }^{14,15,20}$. Desde a década de 1990 , este método é utilizado na Obstetrícia. Vários autores descreveram os índices da artéria oftálmica em pacientes nos diferentes estados hipertensivos durante a gestação ${ }^{9,11-14,23}$. O estudo pioneiro em gestantes foi publicado em 1997, e os autores analisaram o IP da artéria oftálmica de gestantes com hipertensão arterial transitória e hipertensão arterial crônica, encontrando valores mais baixos do IP nessas pacientes ao compará-las ao grupo normal ${ }^{9}$. Estes dados foram confirmados por estudo recente publicado por pesquisadores brasileiros em $2008^{14}$.

O objetivo principal deste estudo foi analisar o comportamento da artéria oftálmica em gestantes portadoras de LES e sem doença renal em atividade em relação às mulheres não-grávidas com LES e às gestantes normais. Não há na literatura nenhum estudo que tenha avaliado se o LES é capaz de produzir modificações na reatividade vascular da artéria oftálmica durante a gravidez. Além disso, não se sabe se o tempo de evolução da doença está associado de modo positivo ou negativo a modificações da anatomia e reatividade vascular desta artéria. Vários estudos descrevem a aplicabilidade da doplervelocimetria da artéria oftálmica para o seguimento de gestantes hipertensas ${ }^{9,13,14,23}$. É necessário saber se este método poderá auxiliar no estudo de outras doenças como, por exemplo, o LES, em que ocorre também o acometimento multissistêmico com importante dano vascular ${ }^{17,18}$.

Os valores descritos do IP, RPV e VDF da artéria oftálmica encontrados no grupo de grávidas normais no atual estudo foram semelhantes a dados já descritos na literatura ${ }^{15,16}$. Há um estudo que estabeleceu o nomograma desses índices da artéria oftálmica nas diferentes idades gestacionais. Os autores concluíram que nenhuma das variáveis analisadas (IR, IP, PVS, RPV, VDF) sofrem alterações durante segundo e terceiro trimestres da gestação $0^{15}$.

Os resultados deste estudo mostraram semelhanças entre as variáveis (IP, VDF e RPV da artéria oftálmica de pacientes lúpicas tanto no grupo de grávidas quanto no grupo de não-grávidas. No entanto, houve redução dos valores IP quando comparados ao grupo de grávidas normais. Isso sugere que o LES exerce pequeno efeito vasodilatador sobre a circulação orbital e central, resultando em aumento de perfusão local. Foi registrada, também, elevação do RPV e da VDF da artéria oftálmica de pacientes lúpicas quando comparadas às grávidas normais. Estes dois índices citados acima são importantes na determinação da impedância arterial à passagem do sangue no território avaliado, sendo que a sua queda define redução de resistência arterial local. A presença do padrão de fluxo de baixa impedância sugere haver priorização de fluxos para o sistema nervoso central, descrito como centralização materna em $2002^{11}$.

Todos os parâmetros analisados da artéria oftálmica em pacientes lúpicas indicaram maior perfusão no território vascular orbital quando comparados a grávidas normais. Entretanto, os valores descritos neste estudo para gestantes com LES são inferiores aos descritos na literatura para as pacientes com pré-eclâmpsia. Dessa maneira, as variáveis Doppler da artéria oftálmica poderão ser aplicadas na tentativa de caracterizar a presença de pré-eclâmpsia em pacientes com LES, tendo em vista as dificuldades encontradas neste diagnóstico diferencial.

Diante da vasculopatia descrita nas pacientes com lúpus, parece incoerente a detecção de sinais de hiperperfusão e vasodilatação da artéria oftálmica. Sabe-se que essa artéria origina-se da artéria carótida interna, principal vaso nutridor do sistema nervoso central. Existe uma conexão vascular importante entre os sistemas carotídeos interno e externo, através de shunt secundário, que pode determinar reatividades vasculares individuais neste território ${ }^{23}$. Mudanças nos calibres das artérias pelos mecanismos de autorregulação dependem de múltiplas variáveis, como mecanismos miogênicos, atividade simpática perivascular, produção endotelial de prostaciclina e óxido nítrico ${ }^{23}$. Acredita-se que a diminuição da impedância na artéria oftálmica observada neste estudo possa decorrer de mecanismos autorregulatórios vasculares, que têm como finalidade manter a oxigenação ao território central, descrito como centralização materna.

Sabe-se que o LES leva a alterações importantes e complexas na microvasculatura sistêmica ao longo do tempo. 
Mulheres jovens portadoras de lúpus têm altas taxas de doenças coronarianas em relação à população normal da mesma faixa etária ${ }^{24}$. A diminuição da complacência arterial e a disfunção endotelial podem antecipar o envelhecimento das artérias. Vários estudos descrevem a presença de aterosclerose subclínica em pacientes com LES, e a patogênese desta alteração não é totalmente entendida. Tem sido proposto que a injúria vascular autoimune promova disfunção endotelial e formação de placas ateromatosas nos vasos ${ }^{25}$.

A complacência arterial é regulada por fatores funcionais e estruturais, incluindo o óxido nítrico derivado do endotélio vascular. A disfunção arterial está associada ao processo inflamatório sistêmico agudo e crônico, que está exacerbado no grupo de pacientes portadoras de LES $^{18}$. Vários estudos já demonstram que as citocinas observadas em pacientes com lúpus podem reduzir a produção do óxido nítrico, bem como alterar sua biodisponibilidade no endotélio arterial ${ }^{26}$.

Os conhecimentos obtidos neste estudo poderão auxiliar no melhor entendimento da fisiopatologia do LES, bem como poderão ser empregados em estudos futuros como método complementar no diagnóstico diferencial entre pré-eclâmpsia e a atividade de doença renal nas gestantes com lúpus.

\section{Referências}

1. Borba EF, Latorre LC, Brenol JCT, Kayser C, Silva NA, Zimmerman $\mathrm{AF}$, et al. Consenso de lúpus eritematoso sistêmico. Rev Bras Reumatol. 2008;48(4): 196-207.

2. Bertsias G, loannidis JP, Boletis J, Bombardieri S, Cervera R, Dostal C, et al. EULAR recommendations for the management of systemic lupus erythematosus. Report of Task Force of the EULAR Standing Committee for International Clinical Studies Including Therapeutics. Ann Rheum Dis. 2008;67(2): 195-205.

3. Ruiz-Irastorza G, Khamashta MA. Managing lupus patients during pregnancy. Best Pract Res Clin Rheumatol. 2009;23(4):575-82.

4. Figueró-Filho EA, Lopes AHA, Senefonte FRA, Duarte G. Lúpus eritematoso sistêmico e gestação. Femina. 2005;33(6):45 1-61.

5. Sibai BM. Imitators of severe preeclampsia. Obstet Gynecol. 2007; 109(4):956-66.

6. Ruiz-Irastorza G, Khamashata MA. Evaluation of systemic lupus erythematosus activity during pregnancy. Lupus. 2004;13(9): 679-82.

7. Belfort MA, Saade GR. Retinal vasospasm associated with visual disturbance in preeclampsia: color flow Doppler findings. Am J Obstet Gynecol. 1993;169(3):523-5.

8. Hata T, Senoh D, Hata K, Kitao M. Ophthalmic artery velocimetry in pregnant women. Lancet. 1992;340(8812):182-3.

9. Hata T, Senoh D, Hata K, Kitao M. Ophthalmic artery velocimetry in preeclampsia. Gynecol Obstet Invest. 1995;40(1):32-5.

10. Hata T, Hata K, Moritake K. Maternal ophthalmic artery Doppler velocimetry in normotensive pregnancies and pregnancies complicated by hypertensives disorders. Am J Obstet Gynecol. 1997; 177(1):174-8.

11. Nakatsuka M, Takata M, Tada K, Kudo T. Effect of nitric oxide donor on the ophthalmic artery flow velocity waveform in preeclamptic women. J Ultrasound Med. 2002;21(3):309-13.

13. Takata $M$, Nakatsuka $M$, Kudo T. Differential blood flow in uterine, ophthalmic, and brachial arteries of preeclamptic women. Obstet Gynecol. 2002;100(5 Pt 1):931-9.

13. Diniz AL, Moron AF, Santos MC, Sass N, Pires CR. Ophthalmic and central retinal arteries Doppler in preeclamptic women with visual disturbance and headache. Ultrasound Obstet Gynecol. $2005 ; 26(4): 462$.

14. Diniz AL, Moron AF, Santos MC, Sass N, Pires CR, Debs CL. Ophthalmic artery Doppler as a measure of severe pre-eclampsia. Int J Gynaecol Obstet. 2008;100(3):216-20.
15. Carneiro RS, Sass N, Diniz AL, Souza EV, Torloni MR, Moron AF. Ophthalmic artery Doppler velocimetry in healthy pregnancy. Int J Gynaecol Obstet. 2008;100(3):2 1 1-5.

16. De Oliveira CA, de Sá RA, Velarde LG, Marchiori E, Netto HC, Ville Y. Doppler velocimetry of the ophthalmic artery in normal pregnancy: reference values. J Ultrasound Med. 2009;28(5): 563-9.

17. Telles RW, Lanna CCD, Ferreira GA, Carvalho MAP, Ribeiro AL. Freqüência de doença cardiovascular aterosclerótica e de seus fatores de risco em pacientes com lúpus eritematoso sistêmico. Rev Bras Reumatol. 2007;47(3): 165-73.

18. Booth AD, Wallace S, McEniery CM, Yasmin, Brown J, Jayne $D R$, et al. Inflammation and arterial stiffness in systemic vasculitis: a model of vascular inflammation. Arthritis Rheum. 2004;50(2):581-8.

19. Hochberg MC. Updating the American College of Rheumatology revised criteria for the classification of systemic lupus erythematosus. Arthritis Reum. 1997;40(9):1725.

20. Diniz ALD, Moron AF, Santos MC, Sass N. Dopplervelocimetria colorida dos vasos orbitais: técnica de exame e anatomia vascular normal. Radiol Bras. 2004;37(4):287-90.

21. Diniz ALD, Moron AF, Santos MC, Sass N, Pires CR. Dopplervelocimetria das artérias oftálmica e central da retina em gestantes normais. Rev Bras Ginecol Obstet. 2005;27(4): 168-73.

22. Report of the National High Blood Pressure Education Program Working Group on High Blood Pressure in Pregnancy. Am J Obstet Gynecol. 2000; 183(1):S1-S22.

23. Belfort MA, Saade GR, Grunewald C, Dildy GA, Varner MA, Nisell $H$. Effects of blood pressure on orbital and middle cerebral artery resistances in healthy pregnant women and women with preeclampsia. Am J Obstet Gynecol. 1999;180 (3 Pt 1):601-7.

24. Kao AH, Sabatine JM, Manzi S. Update on vascular disease in systemic lupus erythematosus. Curr Opin Rheumatol. 2003; 15(5):519-27.

25. Cypiene A, Kovaite M, Venalis A, Dadoniene J, Rugiene R, Petrulioniene $Z$, et al. Arterial wall dysfunction in systemic lupus erythematosus. Lupus. 2009;18(6):522-9.

26. Verma S, Wang CH, Li SH, Dumont AS, Fedak PW, Badiwala $M V$, et al. A self-fulfilling prophecy: C-reactive protein attenuates nitric oxide production and inhibits angiogenesis. Circulation. 2002; 106(8):913-9. 